and for each particular $f$ the measure may be so chosen that (moreover)

$$
\int_{B} \log |f(x)| m_{8}(d x) \geqq \log |f(s)| .
$$

Naturally, if for some reason there is for some $s$ only one measure satisfying 7.12 , then 7.13 holds for that measure.

\title{
BIBLIOGRAPHY
}

I. L. H. Loomis, An introduction to abstract harmonic analysis, New York, 1953.

II. G. Szegö, Über die Randwerte einer analytischen Funktion, Math. Ann. vol. 84 (1921) pp. 232-244.

III. I. Gelfand, D. Raikov, and G. Šilov, Commutative normed rings, Uspehi Matematiceskih Nauk N.S. vol. 2 (1946) pp. 48-146. (In Russian).

IV. D. Milman, Characteristics of extremal points of regularly compact sets, Doklady Akademia Nauk SSSR N.S. vol. 57 (1947) pp. 119-122 (In Russian); Mathematical Reviews vol. 9 (1948) p. 192.

University of California, Los Angeles

\section{FAMILIES OF CURVES}

\section{S. STEIN}

Amasa Forrester in [1] proved the following theorem of a mixed Euclidean and topological character. If $\phi$ is a continuous map without fixed points on the Euclidean $n$-sphere such that $\phi^{2}$ is the identity, then the chords $P \phi(P)$ for all points $P$ of the sphere completely fill the interior of this sphere.

The object of this note is to generalize this theorem to a purely topological statement.

First we recall the definition of retract. If $B C A$ are two spaces, then $B$ is a retract of $A$ if there is $r: A \rightarrow B$ which leaves fixed all points of $B$. (If $X$ and $Y$ are spaces the symbol $f: X \rightarrow Y$ shall denote a continuous map from $X$ to $Y$.)

Let $I$ denote the unit interval. If $F: B \times I \rightarrow A$ and $t \in I$, define $F_{t}: B \rightarrow A$ by $F_{t}(b)=F(B, t)$ for all $b \in B$.

ObServation. If $F: B \times I \rightarrow A$ and if $B$ is a retract of $A$ by the map $r$ and if $p, q \in I$, then $r F_{p}$ is homotopic to $r F_{q}$.

In fact such a homotopy is provided by $G: B \times I \rightarrow B$ defined by

Presented to the Society, November 28, 1953; received by the editors October 26, 1953 and, in revised form, February 28, 1954. 
$G(b, t)=r F(b, p+(q-p) t)$. Clearly $G_{0}=r F_{p}$ and $G_{1}=r F_{q}$.

Now let $E^{n+1}$ be the topological $n+1$ dimensional cell and $S^{n}$ its boundary (an $n$ dimensional topological sphere). Now, for any point $P \in E^{n+1}-S^{n}, S^{n}$ is a retract of $E^{n+1}-\{P\}$ by the map

$$
r: E^{n+1}-\{P\} \rightarrow S^{n}
$$

defined by carrying over the central projection of the Euclidean cell by a homeomorphism. This fact and the observation yield:

Proposition 1. If $f_{i}: S^{n} \rightarrow S^{n}, i=0,1$, are not homotopic and if $F: S^{n} \times I \rightarrow E^{n+1}$ satisfies $F(P, i)=f_{i}(P), i=0,1$, all $P \in S^{n}$, then $F$ is onto $E^{n+1}$.

Proposition 2 (generalization of Forrester's theorem). Let $\phi: S^{n} \rightarrow S^{n}$ be of period $p \neq 1$. Let $F: S^{n} \times I \rightarrow E^{n+1}$ satisfy (a) $F(P, 0)=P$ and (b) $F(P, 1)=F(\phi(P), 1)$. Then $F$ is onto $E^{n+1}$.

Proof. Observe first that it is sufficient to prove this for $p$ prime. For if $p$ were not prime and $q$ is a prime dividing $p$, then the hypothesis of Proposition 2 is satisfied with $\phi$ replaced by $\phi^{p / q}$ and the latter is of prime period. In the following proof therefore $p$ is taken to be prime.

Assume on the contrary that there is a point $Q \in E^{n+1}-F\left(S^{n} \times I\right)$. By (a), $Q \notin S^{n}$ and by a previous remark there is a retraction $r: E^{n+1}$ $\rightarrow\{Q\} \rightarrow S^{n}$. Regarding $F$ as a map into $E^{n+1}-Q$ we would have $r F_{0}$ homotopic to $r F_{1}$. Now $r F_{0}$ is the identity map of $S^{n}$ (hence of degree 1$)$ while $r F_{1}$ has the property that $\left(r F_{1}\right) \phi=r F_{1}$ on account of condition (b).

To conclude the proof it shall be shown that any map $g: S^{n} \rightarrow S^{n}$ satisfying $g \phi=g$ has a degree divisible by $p$.

By [2] there is a cycle of the form $c+\phi(c)+\phi^{2}(c)+\cdots+\phi^{p-1}(c)$ in a generator of $H^{n}\left(S^{n}, J_{p}\right)$. Calling this cycle $z$ we have $g(z)=p c=0$ $(\bmod p)$. Thus the degree of $g$ is divisible by $p$. This concludes the proof of Proposition 2.

Forrester's family of straight lines may be described by

$$
F: S^{n} \times I \rightarrow E^{n+1}
$$

where $F(P, t)$ is the point $Q$ on the line segment joining $P$ to $\phi(P)$ such that $P Q / P \phi(P)=t / 2$.

If the notion of homotopy is translated into the language of a continuous family of curves then Proposition 2 becomes:

Propositron 2'. If (1) $\phi: S^{n} \rightarrow S^{n}$ satisfies the condition stated in Proposition 2 and (2) from each point $P$ of $S^{n}$ there begins one curve of 
$E^{n+1}$ so that the curves beginning at $P$ and $\phi(P)$ have the same terminal point and (3) the parametrization of these curves depend continuously on $P$, then this family of curves fills $E^{n+1}$.

Proposition 3. Let $R^{n}$ refer to $n$ dimensional Euclidean space. If for each direction in $R^{n}$ there is given in a continuous manner precisely one straight line with that direction, then this family of lines fills $R^{n}$.

Proof. Compactify $R^{n}$ to $E^{n}$ by adding two points at infinity for each direction in $R^{n}$. Then apply Proposition 2 or $2^{\prime}$ with $p=2$.

Proposition 4. Let $A$ be a compact subset of $R^{n}$. A necessary and sufficient condition that $A$ be a convex set with the property that each support plane has precisely one contact point is that there exists a continuous choice function on the set of $n-1$ dimensional planes, meeting $A$, with values in $A$. Moreover any such function is onto $A$.

Proof. Let $A$ be a convex subset of $R^{n}$ with the property that each plane of support has one point of contact. Assign to each crosssection its centroid. By Proposition 2 with $p=2$ it is easy to show that this function is onto $A$ (compare to p. 13 of [3]).

The proof of sufficiency is left to the reader. In a subsequent paper the intersection properties of families of curves will be considered.

\section{BIBLIOGRAPHY}

1. Amasa Forrester, $A$ theorem of involutory transformations without fixed points, Proc. Amer. Math. Soc. vol. 3 (1952) pp. 333-334.

2. P. A. Smith, Appendix B, in S. Lefschetz Algebraic topology, Amer. Math. Soc. Colloquium Publications, vol. 27.

3. T. Bonnesen and W. Fenchel, Konvexe Korper, New York, Chelsea, 1948.

University of California, Davis 